so dargestellt und festgelegt haben: »Warum wurden zum Beispiel bestimmte Verhaltensformen als >Wahnsinn gekennzeichnet und klassifiziert, während ähnliche Formen in einem bestimmten historischen Augenblick völlig vernachlässigt wurden; dasselbe gilt für Verbrechen und Kriminalität, dieselbe Frage der Problematisierung gilt für die Sexualität.« (Ebd. [Herv. i. O.]) Diesem Vorgehen nach lassen sich auch weitere Phänomene, darunter auch das Präventionsdispositiv, als Ergebnisse langjähriger Transformationen entschlüsseln, die bis in die Gegenwart ihre Wirkungen haben. Und um ihre Konstruktion verstehen zu können, muss man »eine historische Analyse der Transformation des Diskurses vornehmen, ohne auf das Denken der Menschen, ihre Wahrnehmungsweisen, ihre Gewohnheiten, die Einflüsse, denen sie ausgesetzt waren usw. zu rekurrieren." (Foucault [1969] 2001: 982)

Für das Anliegen einer solchen Geschichte des Denkens als Analyse der Problematisierungen entwickelte Foucault drei Analyseachsen, drei, wie er sie nannte, Brennpunkte der Erfahrung, die ihm ermöglicht haben, sich einem spezifischen Problem zu nähern und ihm eine alternative Auslegung mit den daraus resultierenden gesellschaftspolitischen Konsequenzen anzubieten. Diese Brennpunkte bzw. Analyseachsen werden im Folgenden näher beschrieben.

\title{
2 Foucaults Analyseinstrumente
}

Bei seiner Vorgehensweise hat Foucault seine Analyseinstrumente stets anhand der Forschungsgegenstände entwickelt, die er zu analysieren beabsichtigte. Demnach bilden die drei Analyseachsen eher heuristische Kategorien, die das Analyseverfahren $\mathrm{zu}$ verdeutlichen helfen.

\subsection{Produktion des Wissens}

Foucaults erste Analyseachse konzentrierte sich auf die Produktion und Kanalisierung des Wissens, durch das die Ordnung in der Gesellschaft hergestellt wird. Beeinflusst von Jean Hyppolite und Louis Althusser, Friedrich Nietzsche, Pierre Klossowski, Maurice Blanchot und Georges Bataille (vgl. Foucault [1978] 2005b: 53; auch Kehm 1991: 33) war Foucault daran interessiert, die diskursive Natur des Wissens zu problematisieren und den Einfluss der Produktion von Sinnhaftigkeiten, Bedeutungen und Zuschreibungen auf die subjektive und kollektive Wahrnehmung $\mathrm{zu}$ untersuchen. Gerade in der Wissensproduktion wurzelt seiner Meinung nach das Problem, dass bestimmte Aussagen einen allgemeinen Geltungs- und Wahrheitsanspruch erheben: 
»]ede Gesellschaft hat ihre eigene Ordnung der Wahrheit, ihre sallgemeine Politikı der Wahrheit: d.h. sie akzeptiert bestimmte Diskurse, die sie als wahre Diskurse funktionieren lässt; es gibt Mechanismen und Instanzen, die eine Unterscheidung von wahren und falschen Aussagen ermöglichen und den Modus festlegen, in dem die einen oder anderen sanktioniert werden; es gibt bevorzugte Techniken und Verfahren zur Wahrheitsfindung; es gibt einen Status für jene, die darüber zu befinden haben, was wahr ist und was nicht.« (Foucault [1977] 1978a: 51 [Herv. i. O.])

Somit schlägt Foucault vor, die Wahrheit als »ein Ensemble von geregelten Verfahren für Produktion, Gesetz, Verteilung, Zirkulation und Wirkungsweise der Aussagen« (a.a.O.: 53) zu betrachten und sie in ihrer diskursiven Natur zu analysieren. Den Diskurs definiert er dabei als »die Gesamtheit erzwungener und erzwingender Bedeutungen, die die gesellschaftlichen Verhältnisse durchziehen « (Foucault [1976] 2003a: 164) und die in der Geschichte unterschiedlich verteilt sind. Mehr noch, Foucault behauptete, dass »in jeder Gesellschaft die Produktion des Diskurses zugleich kontrolliert, selektiert, organisiert und kanalisiert wird - und zwar durch gewisse Prozeduren, deren Aufgabe es ist, die Kräfte und die Gefahren des Diskurses zu bändigen, sein unberechenbar Ereignishaftes zu bannen, seine schwere und bedrohliche Materialität zu umgehen.« (Foucault [1970] 1998: 11) Die Analyse der Diskurse und ihre Kritik werden auch deswegen unerlässlich, weil nicht »jedermann Zugang zu den Diskursen hat" (a.a.O.: 26) und daher die Bedingungen ihrer Einsetzung, die Formen ihrer Wirkung und die Regeln ihrer Funktion nicht ändern kann.

Des Weiteren gehorchen Diskurse einer bestimmten Logik bzw. Rationalität, die vorgibt, wie im Diskurs vorgegangen werden soll.

\subsubsection{Die Rationalität(-en)}

Rationalitäten bilden einen Wissensraum, in dem bestimmte Zusammenhänge plausibel erscheinen und andere nicht. Einen Wissensraum zu rationalisieren heißt, Begriffe und Konzepte zu erarbeiten, Gegenstände und ihre Beziehungen untereinander zu spezifizieren, Argumente und Begründungen zu definieren usw. (vgl. Lemke 2000: 32). Eine Rationalität wirkt somit auch politisch, indem sie spezifische Interventionsformen vorgibt und damit Regierungsräume erschließt:

»Eine politische Rationalität ist nämlich kein reines und neutrales Wissen, das die zu regierende Realität lediglich >re-präsentiert‘, sondern stellt selbst bereits eine intellektuelle Bearbeitung der Realität dar, an der dann politische Technologien ansetzen können. Darunter sind Apparate, Verfahren, Institutionen, Rechtsformen etc. zu verstehen, die es erlauben sollen, die Objekte und Subjekte einer politischen Rationalität entsprechend zu regieren.«(Ebd. [Herv. i. O.]) 
Foucault sprach nicht nur von einer singulären Rationalität, die die Geschichte und die Gesellschaft durchdringt, sondern von mehreren Rationalitäten, die »von unterschiedlicher Reichweite und Begründung koexistieren und konfligieren, überlagern und modifizieren sich. An die Stelle der seinen $<$ Wahrheit setzt Foucault mannigfache Wahrheitsspiele mit disparaten Regeln und Strategien.« (Bröckling 2014: 172 [Herv. i. O.]) Die Organisation der Gesellschaft geschieht für ihn somit in einem Regime vielfältiger Diskurse und Rationalitäten, Strategien und Techniken, mit denen »spezifische Diskursformationen, soziale Praktiken und Institutionalisierungen« korrespondieren (ebd.).

Aus der Perspektive der Rationalitäten kann dann auch die Frage nach den Machtverhältnissen (anders) gestellt werden, und zwar »wieso ein bestimmtes Erkenntniselement - sei es wahr oder wahrscheinlich oder ungewiss oder falsch Machtwirkungen hervorbringt und wieso ein bestimmtes Zwangsverfahren rationale, kalkulierte, technisch effiziente Formen und Rechtfertigungen annimmt.« (Foucault [1978] 1992: 31) Um auf diese Fragen antworten zu können, versucht Foucault hinter die allgemeinen Sprach- und Machtstrukturen zu blicken und nach den Bedingungen, unter welchen ein Wahrheitsdiskurs auftaucht, sich rechtfertigt und Machtwirkungen hervorbringt, zu suchen. Das bedeutet, dass er die diskursive Formation eines Wissens- und Wahrheitsregimes aus der Perspektive des Diskursereignisses und der nicht-diskursiven Praktiken zu problematisieren begonnen hat.

\subsubsection{Diskursereignis und nicht-diskursive Praktiken}

Unter einem Diskursereignis versteht Foucault nicht ein Ereignis, das in einer geschriebenen Form festzuhalten wäre. Es handelt sich vielmehr um ein Ereignis,

»das zerstreut ist zwischen Institutionen, Gesetzen, politischen Siegen und Niederlagen, Forderungen, Verhaltensweisen, Revolten und Reaktionen. Eine Mannigfaltigkeit, die man in dem Maße als Diskursereignis erkennen kann und charakterisieren kann, wie sie mehrere Dinge definiert: (i) die Stellung und die Rolle eines Diskurstyps; (ii) die Qualifizierung dessen, der ihn halten soll; (iii) den Cegenstandsbereich, mit dem er sich befasst; (iv) die Art von Aussagen, zu denen er Anlass gibt. Insgesamt ist das Diskursereignis niemals ein Text. Man findet es nicht in einem Text.« (Foucault [1971] 2012: 250)

Bei dieser Form des diskursiven Ereignisses können Brüche und Inkonsistenzen eines Diskurses zum Ausdruck kommen und seine Artikulationsformen und Formationsregeln erkennbar werden. Eine solche methodologische Vorgehensweise, die Foucault unter dem Namen Archäologie zusammenfasste ${ }^{2}$, versucht dann zu deter- 
minieren, wie sich »die Aussagen an der Grenze, die sie von dem Nicht-Gesagten trennt, in der Instanz, die sie beim Ausschluss all der anderen auftauchen lässt « (Foucault [1969] 1981: 173) untersuchen lassen und wie die Formationsregeln, »von denen [ein Komplex von Aussagefakten] abhängt - und die die Positivität charakterisieren, $\mathrm{zu}$ der er gehört - mit nichtdiskursiven Systemen verbunden sein können« (a.a.O.: 231f). Eine kritische Analyse möchte dann nicht zeigen, »wie die politische Praxis den Sinn und die Form des [...] Diskurses bestimmt hat (ebd.), sondern vielmehr, "wie und in welcher Eigenschaft sie zu den Bedingungen seines Auftauchens, seiner Einbeziehung und seines Funktionierens gehört.« (Ebd.)

Die nicht-diskursiven Systeme bilden indessen die äußeren Ausdrucksformen des Diskurses, denn jede diskursive Aussage ist "gleichzeitig nicht sichtbar und nicht verborgen.«(a.a.O.: 158) Wie es Gilles Deleuze weiter präzisiert hat, bedarf es bei einer solchen Art der Problematisierung stets zwischen »dem Sagbaren und dem Sichtbaren, zwischen den diskursiven Formationen und den nicht-diskursiven Formationen, zwischen den Ausdrucksformen und den Inhaltsformen « zu unterscheiden (Deleuze 1992: 71). ${ }^{3}$ Nicht-diskursive Praktiken bilden in diesem Sinne eine Form des Diskurses, die sich in Handlungen, Verhaltensweisen, Entscheidungen und Bewegungen manifestiert und die den Diskurs lebendig und wirkmächtig macht. Die Kopplung diskursiver und nicht-diskursiver Praktiken bekräftigt dann die herrschende politische Rationalität, was dazu führt, dass, wie Judith Butler in Anlehnung an Foucault verdeutlicht hat, "gewisse Verhaltensweisen für den Umgang mit bestimmten Problemen im Lauf der Zeit als ihre Folge eine feste Onto-

nämlich die Aufgabe, »die Diskurse in ihrer Spezifität zu definieren; zu zeigen, worin das Spiel der Regeln, die sie in Bewegung setzten, irreduzibel auf jedes andere ist.« (Ebd.) Nicht die Symbolik oder Kausalität von Ereignissen, sondern die Möglichkeit ihres Erscheinens steht im Vordergrund der Analyse. Wichtig zu bemerken ist, dass diesem analytischen Vorgang »die Instanz des schöpferischen Subjekts als sraison d'être eines Werkes und Prinzip seiner Einheit«fremd ist (ebd. [Herv. i. O.]) und dass es Foucaults Anliegen war, nach der »Cesamtheit von anonymen, historischen, stets im Raum und in der Zeit determinierten Regeln, die in einer gegebenen Epoche und für eine gegebene soziale, ökonomische, geographische oder sprachliche Umgebung die Wirkungsbedingungen der Aussagefunktion definiert haben« (a.a.O.: 171) zu fragen. Eine mögliche Anwendung der archäologischen Vorgehensweise illustriert Foucault am Beispiel der klinischen Medizin (vgl. a.a.O.: 231ff).

Deleuze analysiert die Unterscheidung zwischen den Sichtbarkeiten und Sagbarkeiten, welche Foucault durch seine Vorgehensweise erkennt, folgenderweise: »Eine `Epoche` geht weder den Aussagen voraus, die sie ausdrücken, noch den Sichtbarkeiten, die sie erfüllen. Dies sind die beiden wesentlichen Aspekte: einerseits impliziert jede Schicht, jede historische Formation eine Verteilung des Sichtbaren und des Sagbaren auf sich selbst; andererseits gibt es von einer Schicht zur anderen Variationen dieser Verteilungen, da die Sichtbarkeiten selbst ihre Modalität und die Aussagen ihre Ordnung wechseln. [...] Was Foucault von der Geschichte erwartet, ist diese Bestimmung des Sichtbaren und des Sagbaren in jeder Epoche, die die Verhaltensweisen und die Mentalitäten, die Ideen überschreitet, da sie sie ermöglicht.«(Deleuze 1992: $70 f$ [Herv. i. O.]) 
logie hervorbringen, und dieser ontologische Bereich beschränkt seinerseits unser Verständnis dessen, was möglich ist.« (Butler 2001)

Im Rückblick auf die Wahrheitsansprüche der Diskurse, auf die Wirkungen von Rationalitäten und die nicht-diskursiven Manifestationen lässt sich dann mit Foucault schließen, dass uns die existierende Wissensproduktion von allem Geschehen nur das zu verstehen und wahrzunehmen vorgibt, "was durch das, was aus der Vergangenheit schon sorgfältig behandelt wurde, verständlich gemacht worden ist; durch das, was eigentlich nur darum behandelt wurde, damit der Rest unverständlich wird." (Foucault [1971] 1974: 113) Darum muss nicht nur der brüchige, verschleierte und unbekannte Weg der Wissensproduktion untersucht werden, sondern auch die damit verbundenen nicht-diskursiven Praktiken und die darin beinhalteten Technologien der Macht. Diese müssen einer Kritik unterzogen werden, die sich als »eine fragende Beziehung zum Feld der Kategorisierung selbst konstituiert und sich dabei zumindest implizit auf die Grenze des epistemologischen Horizontes bezieht, innerhalb dessen Praktiken geformt werden.« (Butler 2001) Denn es geht nicht darum, wie Butler meint, die diskursive und nicht-diskursive »Praxis auf einen vorgegebenen epistemologischen Kontext zu beziehen, sondern aus der Kritik genau jene Praxis zu machen, die die Grenzen dieses epistemologischen Horizontes selbst zu Tage bringt und gleichsam dessen Umrisse zum ersten Mal, wie wir sagen könnten, in Beziehung zu seiner eigenen Grenze erscheinen lässt. « (Ebd.) Es handelt sich also um ein Spiel an der Grenze des epistemologischen Feldes, das zum Ziel hat, die Bedingtheit dieses Wissens, seine Wahrheitsansprüche und unsere Beziehung zu der daraus folgenden Regierungspraxis zu verdeutlichen und zu hinterfragen. Somit knüpft die Erforschung der Wissensproduktion an die Untersuchung der Machttechnologien an und leitet über zu der Untersuchung von Foucaults zweiter Analyseachse - der Macht.

\subsection{Technologien der Macht}

Die Behauptung, dass das Wissen und die darin liegenden Wahrheiten diskursiv hervorgebracht werden, eröffnet eine Serie an Fragenstellungen: Wem obliegt das Recht, die Macht anhand dieses Wissens auszuüben? Wieso gilt es gewissen Regeln zu gehorchen? Aufgrund welcher Ermächtigung prävalieren bestimmte Wahrheiten? Welche Wahrheiten werden nicht hinterfragt? Welche Machtregime können durch bestimmte Wissensregime entstehen? Was bedeutet das für die gegenwärtigen Regierungsverhältnisse? Solche und ähnliche Fragestellungen richten den Blick auf die Praxis der Macht; einer Macht, die die Transformation des Wissens in ein »relationale[s] Bezugssystem, in dem Bedeutungen kodiert und Handlungsweisen mit normativen Wertungen aufgeladen werden « begleitet (Mattissek 2008: 39) und innerhalb einer so gewordenen politischen Rationalität eine Regierungsordnung einleitet und aufrechterhält. Die Analyse der Denk- und Wahrheitssysteme, welche 
Foucault anhand unterschiedlicher Diskursformationen vorgenommen hatte, und die Verzweiflung über die Möglichkeiten und Bedingungen, unter welchen solche diskursiven Formationen entstehen konnten, führte ihn zur Hinterfragung des Geflechts von Kräfteverhältnissen, die sich hinter jedem Wahrheitssystem verbergen und es bestimmen.

\subsubsection{Von Kräfteverhältnissen zu Machtbeziehungen}

Schon am Anfang seiner Untersuchungen bezweifelte Foucault die Sinnhaftigkeit einer Analyse, die den Ausdruckswert oder die formalen Transformationen eines Diskurses untersuchen würde und plädierte stattdessen für die Untersuchung ihrer Existenzweisen, d.h. der Weisen ihrer Erscheinung, Funktion und Abhängigkeiten (vgl. Foucault [1969] 2001: 1028). Dem Subjekt eines Diskurses gilt es dann »seine Rolle ursprünglicher Begründung zu nehmen und ihn als variable und komplexe Funktion des Diskurses zu analysieren« (a.a.O.: 1029), um sich folgende Fragen zu stellen: »Welches sind die Existenzweisen dieses Diskurses? Von wo aus wurde er gehalten, wie kann er zirkulieren und wer kann ihn sich aneignen? Welches sind die Plätze, die für verschiedene Subjekte vorgesehen sind? Wer kann diese verschiedenen Subjekt-Funktionen ausfüllen?« (a.a.O.: 1030f) Und es sind die gesellschaftlichen Kräfteverhältnisse, die sich dann in den etablierten Sprecherpositionen und den Möglichkeiten einer Beteiligung und Bestimmung des Diskursstoffes ausdrücken. Einige Jahre später konnte Foucault das Problem noch konkreter postulieren:

»Mein Problem wäre eher folgendes: Welche Rechtsregeln wendet die Macht an, um Diskurse der Wahrheit zu produzieren? Oder weiter: welcher Machttyp vermag Diskurse der Wahrheit zu produzieren, die in einer Cesellschaft wie der unsrigen mit derart mächtigen Wirkungen ausgestattet sind? Damit meine ich folgendes: in einer Gesellschaft wie der unsrigen - im Grunde genommen jedoch in jeder Gesellschaft - wird der soziale Körper von vielfältigen Machtbeziehungen überzogen, charakterisiert und konstituiert, und diese Machtbeziehungen können sich weder auflösen noch stabilisieren noch funktionieren ohne Produktion, Akkumulation, Zirkulation und Funktionieren des Diskurses. Es gibt keine Machtausübung ohne eine bestimmte Ökonomie der Diskurse der Wahrheit, eine Ökonomie, die innerhalb dieses Kräftepaares und von ihm ausgehend funktioniert. Wir sind der Produktion der Wahrheit durch die Macht unterworfen und können die Macht nur über die Produktion der Wahrheit ausüben. Das gilt für jede Cesellschaft.«(Foucault [1976] 1978: 75f)

An dieser Stelle gilt es kurz zwischen den Kräfteverhältnissen und den Machtbeziehungen zu unterscheiden. Die Gesellschaft ist für Foucault von geschichtlich bedingten, wechselseitigen und im Raum zerstreuten Kräfteverhältnissen durchzogen, welche durch Individuen, Gruppen, Klassen usw. verknüpft sind. Diese Ak- 
teure bilden die Knotenpunkte, die verbindenden Elemente, die interagieren und sich stets aufeinander beziehen. In einem solchen »Bündel von Beziehungen « (Foucault [1977] 1978c: 126) wird also jeder mit Potential und Spielraum ausgestattet (vgl. Foucault [1973] 2015: 32), weswegen »jedes Kräfteverhältnis in jedem Augenblick eine Machtbeziehung [impliziert].« (Foucault [1977] 1978b: 112) Deshalb verteilt sich die Macht »über Netze, und in diesem Netz zirkulieren die Individuen nicht nur, sondern sind stets auch in der Position, diese Macht zugleich über sich ergehen zu lassen wie sie auszuüben« (Foucault [1976] 2001: 44), denn »die Macht geht dank des Individuums, welches von ihr konstituiert wurde, durch «(a.a.O.: 45); sie durchdringt es bis zum Innersten.

Des Weiteren weist Foucault darauf hin, dass die Macht »weder einem Jemand noch übrigens einer Gruppe [gehört]; es gibt Macht nur, weil es Streuung, Relais, Geflechte, wechselseitige Stützen, Unterschiede des Potentials, Abstände usw. gibt. In diesem System von Unterschieden, das man wird analysieren müssen, kann die Macht zu funktionieren beginnen.« (Foucault [1973] 2015: 17) Eine solche Vorstellung von Macht schließt jedoch nicht aus, dass den Individuen in diesem System ungleiche Möglichkeiten der Machtausübung und des Widerstands zukommen: »Was den Unterschied ausmacht «, so Foucault, »ist die Position, die von jedem der Gegner eingenommen wird «, also das, was es ihm ermöglicht, »mit herrschaftlichen Wirkungen einen Diskurs zu verwenden, der von allen akzeptiert wird und der nach allen Seiten weiter übermittelt wird.« (Foucault [1976] 2003a: 165) Damit stellt sich die Frage, wie genau die Machtausübung funktioniert und wie sie Herrschaftsverhältnisse installiert.

\subsubsection{Wie die Macht funktioniert}

Im Verlauf seiner Analysen musste sich Foucault mit mehreren Machtbegriffen auseinandersetzen und sich von ihnen abgrenzen. So hat er z.B. dem Marx'schen Verständnis von Macht widersprochen, wonach diese "nach dem Modell der Ware gebildet« sei (Foucault [1976] 2003b: 225), ebenso dem auf das Problem der Souveränität beschränkten juridisch-philosophischen Denken über Macht (vgl. Foucault [1977] 1978b: 109). Stattdessen hat er versucht, das Phänomen der Macht durch andere Attribute aufzugreifen. Das bedeutete für ihn, sich auf den Mechanismus, auf die Funktionsweise und Praxis der Macht, statt auf ihr Wesen und ihren Ursprung zu konzentrieren:

»Ich möchte hier einen anderen Weg zu einer neuen Ökonomie der Machtbeziehungen vorschlagen, der stärker empirisch ausgerichtet und unmittelbarer mit unserer gegenwärtigen Situation verbunden ist, aber auch eine engere Verbindung zwischen Theorie und Praxis impliziert. Dieser neue Forschungsansatz wählt als Ausgangspunkt den jeweiligen Widerstand gegen die verschiedenen Formen von Macht. Oder um es mit einem anderen Bild zu sagen, er benutzt diesen Wider- 
stand als chemischen Katalysator, der die Machtbeziehungen sichtbar macht und zeigt, wo sie zu finden sind, wo sie ansetzen und mit welchen Methoden sie arbeiten. Statt die Macht im Blick auf ihre innere Rationalität zu analysieren, möchte ich die Machtbeziehungen über das Wechselspiel gegensätzlicher Strategien untersuchen.«(Foucault [1982] 2005: 273) ${ }^{4}$

Dort, wo sich ein Widerstand formiert, sind also die Machtverhältnisse sichtbar und analysierbar. Und die Analyse diskursiver Formation ermöglicht gerade diejenigen Stellen aufzudecken, wo widerständige Versuche unternommen werden und wo sich das herrschende Machtsystem um Rechtfertigung und Stabilisierung bemüht. Und weil wir, im Weiteren, »ein geschichtliches Bewusstsein der Situation, in der wir leben [brauchen] « (a.a.O.: 271), versucht Foucault in seinem Forschungsdesign "nicht die Vergangenheit vom Heute aus, sondern das Heute vom Vergangenen aus « zu untersuchen (Visker 1991: 23 zit.n. Michalitsch 2015: 109) und den Pfad der heutigen Regierungs- und Machtkonstellation in seinen geschichtlichen Wandlungen »entsprechend der Intelligibilität der Kämpfe, der Strategien und der Taktiken« (Foucault [1977] 1978a: 29) zu analysieren. Unter solchen Kämpfen und Strategien verstand er »die Gesamtheit der Mittel [...] die eingesetzt werden, um das Funktionieren oder den Bestand eines Machtdispositivs zu sichern." (Foucault [1982] 2005: 292) Die Macht als ein Dispositiv, als ein Ensemble an Praktiken und Strategien zu denken hat ihm dann ermöglicht, in seinen Analysen die Marx'sche »dialektische Logik des Widerspruchs (Rau 2010: 94) und das klassische juridische und negative Analyseraster der Macht zu ersetzen (vgl. Foucault [1977] 1978b: 105). Die Macht denkt Foucault als ein Spiel, als ein Interaktionsmoment ${ }^{5}$, in dem die Kräfteverhältnisse in Bewegung gebracht werden und Machteffekte produzieren. Damit bezeichnet die Machtausübung »keine bloße Beziehung zwischen individuellen oder kollektiven >Partnern<, sondern eine Form handelnder Einwirkung auf andere.«(Foucault [1982] 2005: 285 [Herv. i. O.]) ${ }^{6}$ Und deswegen existiert

Wenn dann Foucault von einer Geschichte des Denkens spricht, so bezieht er sich auf die Geschichte der Technologien und Techniken der Macht (vgl. Foucault [1981] 2005: 230f).

"So wie jeder Mensch ein Bewusstsein hat, das nicht für sich existieren kann, sondern immer nur als ein Bewusstsein >von etwas`, entsteht Macht für Foucault als `strategisches Handeln automatisch dort, wo Menschen in einen Interaktionszusammenhang treten. Dort flottiert sie zwar unabhängig von den einzelnen Subjekten, ist aber - wie das Bewusstsein - nur als ein intentionaler Vorgang erkennbar.«(Pundt 2008: 44 [Herv. i. O.])

6 Am deutlichsten erklärt Foucault diesen Gedanken am Beispiel des Begriffs der Führung: »Der Ausdruck >Führungく (conduite) vermag in seiner Mehrdeutigkeit das Spezifische an den Machtbeziehungen vielleicht noch am besten zu erfassen. >Führung« heißt einerseits, andere (durch mehr oder weniger strengen Zwang) zu lenken, und andererseits, sich (gut oder schlecht) aufzuführen, also sich in einem mehr oder weniger offenen Handlungsfeld zu verhalten. Machtausübung besteht darin, >Führung zu lenken<, also Einfluss auf die Wahrscheinlichkeit von Verhalten zu nehmen.«(Foucault [1982] 2005: 286 [Herv. i. O.]) 
die Macht nur in actu, als eine Praxis und eine auf Handlung anderer gerichtete Handlung (vgl. ebd.). Daraus ließe sich auch Foucaults Behauptung ableiten, dass die Machtverhältnisse der Gesellschaft grundlegend und innewohnend sind: »Ich glaube, dass es keine Gesellschaft ohne Machtbeziehungen geben kann, sofern man sie als Strategien begreift, mit denen die Individuen das Verhalten der anderen zu lenken und zu bestimmen versuchen. (Foucault [1984] 2005b: 899) Damit erstreckt sich die Analyse der Machtbeziehungen auf alle Bereiche, wo Interaktion, Handlung und Führen stattfinden.

\subsubsection{Die Analyse der Machtbeziehungen}

Für Foucault beruht die Macht nicht auf einem Konsens oder einer Gewalt. Diese stellen lediglich »Mittel oder Wirkungen, nicht aber Prinzip oder Wesen der Machtausübung« dar (Foucault [1982] 2005: 286). Das Wesen der Machtausübung besteht vielmehr in den versteckten Spielen, die für Foucault schon auf der kleinsten Ebene des Netzwerks von Machtverhältnissen stattfinden. Aus diesem Grund schlägt er vor, eine "aufsteigende Machtanalyse« (Foucault [1976] 2001: 45) vorzunehmen. Erst durch eine solche Analyse könnte untersucht werden, "wie die Erscheinungen, Techniken, Machtprozesse auf den untersten Ebenen agieren « (a.a.O.: 46) und wie sich diese Verfahren "natürlich verlagern, ausweiten, verändern, aber vor allem, wie sie von globalen Phänomenen besetzt und annektiert werden und wie allgemeinere Machtformen oder wirtschaftliche Vorteile sich in das Spiel dieser zugleich relativ autonomen und unendlich kleinen Machttechnologien einfügen.« (Ebd.)

Von außen her könnten die Machtbeziehungen oft als erstarrt oder verfestigt empfunden werden, eben als Herrschaftsverhältnisse. Tatsächlich weist Foucault darauf hin, dass diese Beziehungen »auf Dauer asymmetrisch sind « (Foucault [1984] 2005b: 891) und dass der Spielraum der Freiheit deswegen »äußerst beschränkt ist." (Ebd.) In dem Verhältnis zwischen Macht und Freiheit ist die Freiheit für Foucault »die Voraussetzung für Macht (als Vorbedingung, insofern Freiheit vorhanden sein muss, damit Macht ausgeübt werden kann, und auch als dauerhafte Bedingung, denn wenn die Freiheit sich der über sie ausgeübten Macht entzöge, verschwände im selben Zuge die Macht und müsste bei reinem Zwang oder schlichter Gewalt Zuflucht suchen). Aber zugleich muss die Freiheit sich einer Machtausübung widersetzen, die letztlich danach trachtet, vollständig über sie zu bestimmen." (Foucault [1982] 2005: 287) Aus diesem Grund gilt es zwischen zwei extremen Formen der Machtausübung zu unterscheiden - den Machtspielen einerseits und den festen Macht- bzw. Herrschaftszuständen andererseits. Und die Analytik der Machtpraktiken bezieht sich auf den Übergang von der einen zu der anderen Form: 
»Mir scheint, dass man unterscheiden muss auf der einen Seite zwischen Machtbeziehungen als strategischen Spielen zwischen Freiheiten, also Spielen, in denen die einen das Verhalten der anderen zu bestimmen versuchen, worauf die anderen mit dem Versuch antworten, sich darin nicht bestimmen zu lassen oder ihrerseits versuchen, das Verhalten der anderen zu bestimmen, und auf der anderen Seite Herrschaftszuständen, die das sind, was man üblicherweise Macht nennt. Und zwischen beiden, zwischen den Spielen der Macht und den Zuständen der Herrschaft, gibt es die Regierungstechnologien, wobei dieser Ausdruck einen sehr weitgefassten Sinn hat: das ist sowohl die Art und Weise, wie man Frau und Kinder leitet, als auch die, wie man eine Institution führt. Die Analyse dieser Techniken ist erforderlich, weil sich häufig mit ihrer Hilfe die Herrschaftszustände errichten und aufrechterhalten.«(Foucault [1984] 2005b: 900)

Da die Macht »auf der Grundlage von bereits bestehenden Differenzierungen operiert « (Jessop 2005: 22) und die Machtspiele ein fester Bestandteil der Gesellschaft sind, besteht das Analyseproblem einerseits darin, zu wissen, »wo sich Widerstand formieren kann« (Foucault [1984] 2005b: 891) und wo die Situation eines ökonomischen, sozialen, institutionellen oder sexuellen Herrschaftszustands umgekehrt werden kann (vgl. ebd.) und andererseits darin, zu wissen, wie man bei den alltäglichen und unumgänglichen Praktiken der Macht »bei denen die Macht sich nicht nicht ins Spiel bringen kann und in denen sie nicht an sich selbst schlecht ist, Herrschaftseffekte vermeiden kann.« (a.a.O.: 899) ${ }^{7}$ Letztendlich führt die Hinterfragung der Herrschaftsverhältnisse notwendigerweise zur Erfindung neuer politischer Strategien, die es erlauben, die operierenden Kräfteverhältnisse »zu modifizieren, und sie in der Weise zu koordinieren, dass diese Modifikation möglich wird und sich in die Realität einschreibt.«(Foucault [1977] 1978b: 113) Wie sich diese Einschreibung vollzieht und wo die Herrschaftseffekte antastbar werden, erforschte Foucault mithilfe seiner dritten Analyseachse - des Subjekts.

7 Die Macht, wie von Foucault mehrmals betont, liegt nicht in den Händen einiger wenigen Akteure. Jeder, der in eine konkrete Handlung eintritt, tritt zugleich in komplexe Spiele, in denen versucht wird, auf die Handlungen anderer einzuwirken. Wie Foucault in seinen Ausführungen zur ethischen Arbeit an sich selbst zeigt, ist es schließlich die Selbstbemeisterung, die Macht über sich selbst, die auch die Macht über die anderen regulieren kann (vgl. Foucault [1984] 2005b: 885). Deshalb legt er einen besonderen Wert auf die Selbstbeherrschung, denn diese »umschließt gewissermaßen den zutiefst asymmetrischen Charakter der Herrschaftsbeziehung, die man mit den anderen hat. Man muss die Herrschaft, die man über die anderen hat, durch die Selbstbeherrschung begrenzen.«(Foucault [1981] 2016: 342) 


\subsection{Die Konstitution des Subjekts}

Die Untersuchung der Wissensproduktion und der darin wirkenden Machtpraktiken mündeten in das umfassende Thema von Foucaults wissenschaftlicher und akademischer Arbeit - der Konstitution des Subjekts (vgl. Foucault [1982] 2005: 269f). So bildeten die unterschiedlichen Formen der Unterwerfung (der Subjektivierung), die immanenten und sich wieder neu erzeugenden Praktiken der (An-)Führung zur Selbstführung (zur Subjektwerdung) und die diesen Praktiken unterliegenden Rationalitäten (die die Subjektmodi erzeugen) im tiefsten den Kern seiner Tätigkeit. Vor allem die Frage, wie das Individuum dazu gelangt, sich selbst als Subjekt gewisser Verhältnisse zu konstituieren, steht im Mittelpunkt seiner Analysen.

Für Foucault hat die Vorstellung von dem Menschen als dem Erzeuger des Wissens und dem Gegenstand eben dieses Wissens das anthropologisch-humanistische Denken des 19. Jahrhunderts geprägt (vgl. Foucault [1969] 1974: 16). Doch in dem Moment, wo die strukturalistischen Ansätze des 20. Jahrhunderts aufgezeigt haben, dass der Mensch »in Strukturen eingebettet ist, d.h. in eine formale Gesamtheit von Elementen, die beschreibbaren Relationen unterworfen sind, hört der Mensch sozusagen auf, das Subjekt seiner selbst zu sein, zugleich Subjekt und Objekt zu sein.« (Foucault [1969] 1974: 16) Damit lehnte Foucault den Versuch ab, "die Zweideutigkeit des Menschen als Subjekt und Objekt « zu hypothesieren (Rau 2010: 113) und konzentrierte sich stattdessen auf das Subjekt als einen Fluchtpunkt von diskursiven und nicht-diskursiven Praktiken, durch welche es sich wahrnimmt und an der Machtpraxis teilnimmt:

»Ich versuche, im Rahmen eines so genau wie möglich abgesteckten historischen Feldes das Ensemble der vorhandenen Praktiken des Subjekts, wie sie sich von der griechisch-römischen Epoche bis heute entwickelt haben, zu bestimmen. Ich glaube, dass wir sowohl die Humanwissenschaften (sofern wir dieses Wort verwenden wollen) als auch insbesondere Psychoanalyse nur verstehen können, wenn wir die Geschichte der Beziehungen zwischen Subjekt und Wahrheit vom Standpunkt dessen aus aufnehmen, was ich, grob gesagt, die Techniken, Technologien, Praktiken usw. nenne, die jene Beziehungen geknüpft und normiert haben.«(Foucault [1982] 2009: 239)

Damit soll nicht die ontologische Natur des Subjekts, seine Existenzbegründung oder seine Autonomie reflektiert werden (vgl. Kelly 2009: 82ff). Vielmehr war es Foucault wichtig, die Analysemöglichkeiten der Subjektwerdung zu erforschen. Und diese ließen sich nur im Zusammenhang mit den zwei bereits erwähnten Brennpunkten der Erfahrung - Wissen und Macht - vollziehen, da sie erst in der gegenseitigen Artikulation sichtbar und analysierbar werden (vgl. Foucault [1983] 2009: 15). Wenn das Subjekt z.B. »in Produktionsverhältnisse und Sinnbeziehungen 
eingebunden ist, dann ist es zugleich auch in hochkomplexe Machtbeziehungen eingebunden « (Foucault [1982] 2005: 270), und für die Analyse solcher Beziehungen »fehlten bislang die entsprechenden Werkzeuge.«(Ebd.) Foucaults Interesse an dieser Forschungslücke erweckte vor allem der wissenschaftliche Aufschwung, mit dem neue Kenntnisse über den Menschen, seinen Organismus und Körper, sein Verhalten und Denken entstanden und ihn zum Objekt der Untersuchung und Unterwerfung zugleich machten. Diese Kenntnisse, dieses Wissen also, gemeinsam mit den Praktiken der Unterwerfung, der Macht, entwickelten sich über eine längere Zeit, weshalb Foucault eine Geschichte der Subjektwerdung schreibt und diese als ein Ereignis des Denkens entziffert, d.h. als einen Moment der historischen Erscheinung, ab dem sich in unserem gesellschaftlichen Denken und in unserer gesellschaftlichen und kulturellen Wahrnehmung eine gewisse Erfahrung einschreibt, die die heutige Seins- und Denkweise prägt und die geläufigen Subjektmodi fundiert. ${ }^{8}$

\subsubsection{Das Ereignis des Denkens in der Geschichte der Subjektivierung}

Dem Ereignis des Denkens kommt in Foucaults Analyse eine wichtige Bedeutung zu. Er selbst hat versucht ein Regime des Zum-Ereignis-Machens (franz. événementalisation) zu etablieren. Dieses wissenschaftliche Vorgehen besteht in zwei Schritten.

Zum einen lädt Foucault dazu ein, einen Bruch mit den Evidenzen vorzunehmen, »denjenigen Evidenzen, auf denen unser Wissen basiert, unser Konsens, unsere Praktiken.« (Foucault [1978] 2005a: 29) Einen möglichen Vorgang hat Foucault folgenderweise illustriert:

»Dort wo man versucht wäre, sich auf eine historische Konstante zu beziehen oder auf ein unmittelbar anthropologisches Merkmal, oder auch auf eine Evidenz, die sich allen auf gleiche Weise aufdrängt, geht es darum, eine >Singularität ‘ auftreten zu lassen. Zu zeigen, dass es gar nicht so ınotwendig war, dass...; dass es gar nicht so evident war, die Wahnsinnigen als Geisteskranke zu betrachten; dass es gar nicht so evident war, dass das Einzige, das man mit einem Delinquenten tun

Am Beispiel der altgriechischen Sorge um sich als einer gesamtkulturellen Erscheinung illustriert Foucault die Geschichte der Subjektwerdung als ein Ereignis des Denkens, welches bis zur heutigen Zeit Auswirkung hat: »Was ich Ihnen in diesem Jahr aufzeigen möchte, ist genau jene Ceschichte, die dazu geführt hat, dass diese gesamtkulturelle Erscheinung (Aufforderung zur Sorge um sich, allgemeine Akzeptanz des Crundsatzes) eine für die hellenistische und römische Gesellschaft (jedenfalls für deren Elite) charakteristische Erscheinung und zugleich ein Ereignis im Denken war. Mir scheint, dass die Herausforderung, auf die sich jede Ceschichte des Denkens einzulassen und die sie zu ihrem Gegenstand zu machen hat, darin besteht, jenen entscheidenden Moment einer kulturellen Erscheinung gegebenen Ausmaßes zu erfassen, der sich in dieser Ceschichte des Denkens dadurch auszeichnet, dass auch noch unsere moderne Seinsweise als Subjekt davon beeinflusst wird. (Foucault [1982] 2009: 
konnte, darin bestand, ihn einzusperren; es war nicht so evident, dass die Ursachen der Krankheit in der individuellen Untersuchung des Körpers zu ermitteln waren usw.« (ebd. [Herv. i. O.])

Zum anderen besteht dieser Vorgang darin, »die Zusammenhänge, die Zusammentreffen, Unterstützungen, Blockaden, Kraftspiele, Strategien usw. wiederzufinden, die zu einem bestimmten Zeitpunkt dasjenige formierten, das anschließend als Evidenz, Universalität oder Notwendigkeit fungieren sollte.« (a.a.O.: 30) Dieser Prozess führt dann, seiner Meinung nach, zu einer kausalen Demultiplikation, einer Zergliederung der Elemente, die das Ereignis ermöglicht und gebildet haben. Diese Zergliederung besteht darin,

»das Ereignis den vielfältigen Prozessen entsprechend zu analysieren, die es konstituieren. So bedeutet die Praxis der Strafhaft als >Ereignis`zu analysieren (und nicht als institutionelle Tatsache oder als ideologischen Effekt), die Prozesse der Pönalisierung (das heißt der schrittweisen Eingliederung in die Form gesetzlicher Bestrafung) der früheren Praktiken der Einsperrung zu definieren; die Praktiken der `Karzeralisierung « der Praktiken der Strafjustiz (das heißt die Bewegung, durch die die Einsperrung als Form der Bestrafung und als Technik der Besserung zu einem zentralen Bestandteil der Pönalität wurde); diese massiven Prozesse müssen selbst zergliedert werden: Der Prozess der Pönalisierung der Einsperrung ist seinerseits aus vielfältigen Prozessen gebildet wie der Konstituierung geschlossener pädagogischer Räume, die auf der Basis von Belohnung und Bestrafung funktionieren usw.« (ebd. [Herv. i. O.])

Mit diesem Vorgang grenzt sich Foucault von den strukturalistischen und historiografischen Methoden $\mathrm{ab}$. An die Stelle der formalen Strukturen und Zeichen des Strukturalismus setzt er »das >Ereignis` und die >Serieく; anstelle historiographischer Kausalitätsbezüge und Kontinuitäten analysiert er die Zerstreuung und Serialität der Erscheinungen (vgl. Dosse 1998: 292f). Insbesondere die Ablösung von einem Denken in Kontinuitäten, welches >das Hereinbrechen der Ereignisse durch ein Denken in der Ungleichzeitigkeit von Ereignisschichten sauszulöschen scheint< (Foucault [1969] 1973: 13), lässt das >Ereignis` und die `Serieく an die Stelle von >Ursprung< und >Bedeutung< treten (vgl. Foucault [1968] 2001: 897; [1969] 1973: 16f).«(Siebenpfeiffer 2008: 250 [Herv. i. O.] $)^{9}$ durch den Begriff des Ereignisses geprägt hat, entstanden auch weitere Grundbegriffe wie die Serie, die Regelhaftigkeit und die Möglichkeitsbedingung. Wie diese Begriffe zueinander stehen, zeigen die Diskursanalytiker folgenderweise: »Wenn ein epistemisches Element (enoncé) in einer diskursiven Umgebung, also in einer Äußerung spontan und unvorhergesehen auftritt, erscheint es als >Ereignis«. Wenn solche Ereignisse häufiger auftreten und damit zu Keimzellen diskursiver Formationen werden, bilden sich `Serien«. Durch die Verdich- 
Die allmähliche Zergliederung der jeweiligen Ereignisse in die kleinsten Einheiten lässt dann auch die Funktionsweise der Mikromächte erscheinen, weshalb Foucault eine aufsteigende Machtanalyse vornehmen wollte. Diese Analyse geht von den kleinsten Mechanismen aus, »die ihre eigene Geschichte, ihren eigenen Weg, ihre eigene Technik und Taktik haben « (Foucault [1976] 2001: 45f) und möchte erhellen, »wie diese Machtmechanismen, die ihre Stabilität und in gewisser Weise ihre eigene Technologie haben, von immer allgemeineren Mechanismen und globaleren Herrschaftsformen besetzt, kolonisiert, verwendet, umgebogen, transformiert, verlagert und ausgedehnt wurden und immer noch werden.« (Ebd.)

Es handelt sich im Weiteren um Mechanismen, die in das Individuum eindringen, seinen Körper kolonisieren, sein Verhalten strukturieren und seine Denkweise beeinflussen. Da das menschliche Individuum immer breiter und tiefer wissenschaftlich erfasst wird, und die neurogenetischen und nanotechnologischen Entwicklungen bezeugen diesen Trend wohl am deutlichsten, dringen auch die Technologien der Macht in immer kleinere und intimere Teile des Individuums ein, ohne von seiner Vorstellung übernommen zu werden (vgl. Foucault [1977] 1978b: 108f). ${ }^{10}$ Solche, »durch Machteffekte als Subjekte konstituierten Körper« (Foucault [1976] 2001: 44) analysiert Foucault gerade anhand der Subjektivierungsthese. Hier hat der Begriff des Subjekts für ihn zwei Bedeutungen:

»Es bezeichnet das Subjekt, das der Herrschaft eines anderen unterworfen ist und in seiner Abhängigkeit steht; und es bezeichnet das Subjekt, das durch Bewusstsein und Selbsterkenntnis an seine eigene Identität gebunden ist. In beiden Fällen suggeriert das Wort eine Form von Macht, die unterjocht und unterwirft." (Foucault [1982] 2005: 275)

Sein Subjektbegriff bietet einen Analysezugang, um die Formen der Unterwerfung sichtbar zu machen, die durch andere Analyseverfahren nicht abgedeckt werden können: »Ich musste eine bestimmte Theorie sa priori< des Subjekts zurückweisen,

tung von Serien diskursiver Ereignisse etablieren sich neue diskursive Strukturen und bilden so >Regelhaftigkeit«. Etablierte diskursive Formationen wirken als >Möglichkeitsbedingungen prägend auf zukünftige diskursive Ereignisse, steuern also Erwartbarkeitszwänge oder -ausschlüsse für künftige Äußerungen.« (Fraas \& Klemm 2005: 3 [Herv. i. O.])

10 Schon in seinen früheren Untersuchungen zu Praktiken der Psychiatrisierung hat Foucault auf die Gefahren der Ermächtigung des menschlichen Körpers hingewiesen: »Tatsächlich ist das Individuum das Resultat von etwas, das ihm vorausgeht, nämlich jenes Mechanismus, all jener Verfahren, welche die politische Macht am Körper festmachen. Weil der Körper ssubjektivisiert ‘ worden ist, das heißt, sich die Subjekt-Funktion an ihm festgemacht hat, weil er psychologisiert worden ist, weil er normalisiert worden ist; deswegen ist etwas wie das Individuum zutage getreten, etwas, worüber man sprechen kann, worüber man Abhandlungen verfassen, worüber man versuchen kann, Wissenschaften zu begründen.« (Foucault [1973] 2015: 91 [ [Herv. i. O.]) 
um diese Analyse der Beziehungen zwischen der Konstitution des Subjekts oder verschiedener Formen des Subjekts und den Spielen der Wahrheit, den Praktiken der Macht usw. vornehmen zu können. «(Foucault [1984] 2005b: 888 [Herv. i. O.]) In dieser Hinsicht führte Andrea Bührmann die Analyse ein Stück weiter, indem sie zwischen einer Subjektivierungsweise, d.h. einer Art und Weise, »wie Menschen sich selbst und andere auf einer empirisch faktischen Ebene wahrnehmen, erleben und deuten « (Bührmann 2012: 146) und einer Subjektivierungsform, d.h. einer Art und Weise, »wie Menschen auf einer normativ programmatischen Ebene über bestimmte Praktiken oder Programme lernen sollen, sich selbst und andere wahrzunehmen, zu erleben und $\mathrm{zu}$ deuten « (ebd.) zu unterscheiden begonnen hat. Somit stellt sich erneut die Frage, inwiefern die Individuen in der Lage sind, den Stand der Subjektivierung, d.h. der Unterwerfung, wahrzunehmen und zu erkennen.

\subsubsection{Subjektivierung und Identitätszuweisung}

Foucaults Subjektivierungsthese richtet den analytischen Blick auf die Verfahren, "durch die das Individuum dazu gelangt, sich selbst als Subjekt zu konstituieren« (Foucault [1983] 2009: 18) und verweist damit gleichzeitig auf eine Machtform, die »die Individuen in Kategorien einteilt, ihnen ihre Individualität zuweist, sie an ihre Identität bindet und ihnen das Gesetz einer Wahrheit auferlegt, die sie in sich selbst und die anderen in ihnen zu erkennen haben. (Foucault [1982] 2005: 275) Bei der Identitätsbindung und Individualisierung handelt es sich Foucault nach um Machttechniken, die das Individuum und seinen Lebensraum berauben, es durch Subjektzuweisung unterjochen und seine Freiheit einschränken, was er anhand der Untersuchung von Disziplinierungstechniken und Pastoralmacht ausführlich beschrieben hat. ${ }^{11}$

Seit den letzten Jahrhunderten haben sich diese Techniken der Macht in einer gegenseitigen und wechselhaften »Ko-Formierung von modernem souveränen Staat und modernem autonomen Subjekt « entfaltet (Lemke 2000: 32f) und dieser »fortschreitende[n], gewiss zerstückelte[n], aber dennoch kontinuierliche[n] Verstaatlichung« (Foucault [1984] 2000: 69) müssen wir mit einer Suche nach neuen Formen von Subjektivität entgegnen und damit »die Art von Individualität zurückweisen, die man uns seit Jahrhunderten aufzwingt.« (Foucault [1982] 2005: 280) An dieser Stelle beginnt Foucaults Versuch, die Möglichkeiten der Ermächtigung und der Ent-Subjektivierung zu erforschen und sie als eine ethisch-politische Aufgabe aufzugreifen. Foucault ruft damit zu einer Operation des widerständigen Denkens,

Zur Entwicklung der Disziplinarmacht siehe vor allem Sitzungen vom 7., 14., 21. und 28. November der 1973-1974er Vorlesungsreihe am Collège de France (Foucault [1973] 2015]). Die Geschichte der Pastoralmacht wird in den Sitzungen vom 15. und 22. Februar und vom 1. und 8 März der 1977-1978er Vorlesungsreihe an demselben Institut besonders ausführlich nahegelegt (Foucault [1978] 2006). 
die sich nicht von der Angst omnipräsenter Machtpraktiken, sondern von der Neugier, »die es erlaubt, sich von sich selbst zu befreien« (Foucault [1983] 2005: 664) führen lässt. Dadurch verleiht er auch der philosophischen Aktivität eine emanzipatorische Aufgabe: »Doch was ist dann die Philosophie heute - ich meine die philosophische Aktivität -, wenn nicht die kritische Arbeit des Denkens an sich selbst? Und besteht sie nicht darin, anstatt das zu legitimieren, was man bereits weiß, herauszufinden versuchen, wie und bis wohin es möglich wäre, anders zu denken?«(Ebd.)

\subsubsection{Doppelter Charakter der Subjektivierung}

Mit seinen Untersuchungen zu den Strategien, Verfahren und Techniken, durch die eine Regierungsordnung etabliert wird, eröffnete Foucault den Raum für eine kritische Reflexion, die das Individuum nicht »nur als Rechtssubjekt ins Auge fassen [kann]« (Foucault [1984] 2005b: 901), sondern die »die Freiheit des Subjekts und die Beziehung zu anderen geltend « macht (ebd.). Darin entfaltet sich die doppelte Charakteristik der Subjektivierung, die, einerseits, als ein Prozess der Unterwerfung, und, andererseits, als eine an sich vorgenommene Arbeit verstanden werden kann. Das heißt, dass sich in dem zweiten Fall das Subjekt »anhand der Selbsttechniken, anstatt durch die Unterdrückungstechniken (Macht) oder die diskursiven Techniken (Wissen) [konstituiert] (Gros in Foucault [1982] 2009: 623) ${ }^{12}$ _ Selbst-statt Fremdunterwerfung also. Es ist eine bedeutende Wende in Foucaults Denken, die, nichtdestotrotz, auf eine kontinuierliche und systematische Arbeit an den Techniken des Widerstandes und der Emanzipation hinweist. Diese gründen in dem kritischen und ethisch-politischen Vorhaben, »das Subjekt mit einer Wahrheit auszurüsten, die es nicht bereits erkannte und die nicht bereits in ihm vorhanden war. Es geht darum, aus der gelernten, dem Gedächtnis einverleibten und schrittweise in Anwendung gebrachten Wahrheit ein Quasi-Subjekt zu machen, das souverän in uns herrscht.« (a.a.O.: 610f)

Das abendländische Denken ist für Foucault seit der Antike durch den Imperativ des Erkenne dich selbst! geprägt und dieser lässt sich in einer allgemeineren und expliziten Form als eine Frage folgenderweise postulieren: »Was soll man aus

12 Dem Subjektbegriff bei Foucault wird von den Kritikern vor allem die Verleugnung universalistischer, schöpferischer und historisch-kontinuierlicher Charaktereigenschaften vorgeworfen (vgl. Cohen 2012), wie auch sein unklares und schwammiges Subjektverständnis (vgl. Thiel 2017). In einem seiner letzten Interviews äußerte sich Foucault zu diesem Thema besonders prägnant und unzweideutig (vgl. Foucault [1984] 2007: 283). Das Subjekt oder, genauer gesagt, die Subjektivität sind in seinem Verständnis Begriffe, die eine geschichtliche Analyse der Selbsttechnologien ermöglichen und eine Schablone anbieten, durch die »die Gesamtheit der Subjektivierungsprozesse, denen die Individuen unterworfen waren oder die sie gegenüber sich selbst zum Tragen gebracht haben«(Foucault [1981] 2016: 364) überhaupt erst erfassbar wird. 
sich selbst machen? Welche Arbeit soll man an sich verrichten? Wie soll man sich mittels Handlungen sregieren (se gouverner)<, bei denen man selbst das Ziel, der Anwendungsbereich, das verwendete Instrument und das handelnde Subjekt ist? « (Foucault [1981] 2016: 377f [Herv. i. O.]) Für Foucault besteht darin die »Verehrung oder der Kult, die man sich selbst gegenüber schuldig ist « (Foucault 1982 in Gros 2009: 660) und zwar die Arbeit an einem Selbst, an dem Geschick, das sich aus dem eigenen Leben machen ließe. Wie bereits in der antiken Praxis des Selbst, ist zu dieser Veränderung ein Lehrmeister notwendig, dessen Rolle auch heutzutage unerlässlich ist (in Form einer Fitnesstrainerin, eines Business Coaches, eines geistlichen Begleiters usw.). Das Subjekt ist, heute wie damals, in der Unwissenheit, Lasterhaftigkeit oder Missbildung befangen und seine Befreiung muss es, laut Foucault, nicht durch ein Wissen anstreben, sondern durch die Erlangung eines Zustands, »den es nie, zu keinem Zeitpunkt seines Daseins, gekannt hat. Es hat den Zustand des Nicht-Subjekts durch den des Subjekts zu ersetzen, der durch die volle Entfaltung der Beziehung zu sich selbst definiert ist.« (Foucault [1982] 2009: $169)^{13}$

Mit dieser Aufforderung gipfelt Foucaults langjähriges Werk, das wegen seinem frühen Tod im Jahr 1984 beenden war. Die hinterlassenen analytischen Mittel und Verfahren inspirieren zu einer radikalen Hinterfragung eingefahrener Denkschemen und bieten eine Möglichkeit an, die Spielräume der Macht aufs Neue zu eröffnen und damit die Vorstellung von der existierenden (Regierungs-)Ordnung zu überdenken. Darauf weist vor allem sein Denken über das Regieren und seine Vorstellung des Politischen hin, die im Konzept der Gouvernementalität enthalten sind.

\section{Gouvernementalität - ein Denken über das Regieren}

Die beschriebenen analytischen Vorgänge ermöglichen einen Blick in Foucaults Werkzeugkasten. Foucaults Denken und seine Verfahren haben Eingang auch in die politikwissenschaftlichen Untersuchungen gefunden. Vor allem sein Denken über die Regierungstechnologien, die er im Begriff der Gouvernementalität subsummiert hat, inspirierte einen ganzen Forschungsbereich - die GouvernementalitätsStudien -, der sich mit den Fragen des Regierens und Regiert-Werdens befasst.

13 Am Beispiel der Homosexualität offenbart Foucault sein Interesse, Neues zu erzeugen, statt sich in bekannte Modelle einzureihen. Deshalb wollte er nicht wissen: »Wer bin ich? Was ist das Geheimnis meines Begehrens?« (Foucault [1981] 1985: 85), sondern vielmehr: »Was für Beziehungen können über die Homosexualität aufgebaut, entworfen, erweitert und von Fall zu Fall verschieden gestaltet werden?« (ebd.) 